\title{
Germanica
}

\section{Snobismus und neue deutsche Popliteratur am Beispiel von Benjamin von Stuckrad-Barre}

Snobbery in German Pop-Literature: Benjamin von Stuckrad-Barre.

Benjamin von Stuckrad-Barre et le snobisme de la nouvelle littérature pop en

Allemagne.

Alfred Strasser

\section{(2) OpenEdition}

Journals

\section{Édition électronique}

URL : http://journals.openedition.org/germanica/1422

DOI : 10.4000/germanica.1422

ISSN : 2107-0784

Éditeur

Université de Lille

\section{Édition imprimée}

Date de publication : 31 décembre 2011

Pagination : 135-144

ISBN : 9782913857285

ISSN : 0984-2632

\section{Référence électronique}

Alfred Strasser, «Snobismus und neue deutsche Popliteratur am Beispiel von Benjamin von StuckradBarre », Germanica [Online], 49 | 2011, Online erschienen am: 26 März 2012, abgerufen am 06 Oktober 2020. URL : http://journals.openedition.org/germanica/1422 ; DOI : https://doi.org/10.4000/ germanica. 1422

Ce document a été généré automatiquement le 6 octobre 2020.

(c) Tous droits réservés 


\section{Snobismus und neue deutsche Popliteratur am Beispiel von Benjamin von Stuckrad-Barre}

Snobbery in German Pop-Literature: Benjamin von Stuckrad-Barre. Benjamin von Stuckrad-Barre et le snobisme de la nouvelle littérature pop en Allemagne.

Alfred Strasser

Benjamin von Stuckrad-Barre gilt mit Andreas Mand, Thomas Brusig, Thomas Meinecke, Rainald Goetz, Marc Fischer, Max Gold, Christian Kracht und anderen als einer der Hauptvertreter der neuen deutschen Popliteratur ${ }^{1}$, einer literarischen Strömung, die ab der Mitte der neunziger Jahre des vorigen Jahrhunderts im deutschen Literaturbetrieb eine wesentliche Rolle spielte. Den Texten dieser meist nach 1965 geborenen Autoren warf die Literaturkritik mehrheitlich fehlende künstlerische Qualität vor. Im Gegensatz dazu nahmen die meist jugendlichen Leser diese Literatur begeistert auf und feierten ihre Autoren wie Pop-Stars. Einen Grund für die fast durchweg schlechten Kritiken sieht der Literaturwissenschaftler Moritz Baßler darin, dass diese Literatur - zumindest vordergründig - Sozialkritik oder gar politisches Engagement vermissen lasse : „Diese Prosa hatte keine Tiefenstruktur, sie war nicht auf Entlarvung eines schönen Scheins hin angelegt, sie war nicht von einem gesellschaftlichen oder sonstigen Problem her geschrieben und lief nicht auf ein Coming out hinaus. “2

Die Abkehr der Autoren der neuen Popliteratur von allem Politischen - sie unterscheidet sich dadurch wesentlich von der Popliteratur der sechziger Jahre etwa eines Rolf Dieter Brinkmann - geschieht jedoch nicht aus reinem Desinteresse an der Politik, sondern sie ist, zusammen mit ihrem demonstrativ vorgetragenen Konservatismus, als eine Protesthaltung gegenüber einer überpolitisierten Vorgängergeneration zu bewerten. Insofern ihre Haltung sich in erster Linie über die 
dezidierte Abgrenzung gegenüber etablierten Positionen definiert, ist sie mit Fug und Recht snobistisch zu nennen.

3 Snob ist zuerst, nach William Thackeray ${ }^{3}$, vor allem die Bezeichnung für einen Aufsteiger, der den Geschmack der Oberschicht auf vulgäre Art imitierte, gleichzeitig verachtet er die Mittelschicht, von der er sich abgrenzen will und legt ihr gegenüber ein überhebliches Verhalten an den Tag. Thackeray beschränkt den Begriff aber nicht nur auf diese Gruppe, er spricht auch von "The Snob Royal“ und meint damit James I. ${ }^{4}$ und Louis XIV. von Frankreich. Bekannt wurde der Begriff „Snob“ schließlich durch Die Snobs, Prosaskizzen (The Book of Snobs 1848) $)^{5}$. Thackeray charakterisiert Snobs einerseits als „hochmütig, brutal, dumm und völlig selbstsicher", andererseits können sie aber auch „arm, staunend, kniend und einfältig“" sein. Der Begriff Thackerays wurde schnell aufgegriffen und erfuhr sehr bald einen Bedeutungswandel: Snobismus stand bald auch für verschiedene Formen der Verengung des Daseins, womit der Snob zunächst als Ausgeburt des Philistertums (Schopenhauer) oder gar als der Bourgeois par excellence erscheint. So wird im Laufe der Kulturgeschichte der Snob zu demjenigen, der sich über die Allgemeinheit erhebt, indem er das Besondere für sich vindiziert, mit dem er sich dezidiert von eben diesen Philistern und Bourgeois abzusetzen sucht. Sine nobilitate vindiziert er den „Adel“ des Neuen und Extravaganten, rechnet sich einer missverstandenen „avant-garde“ zu und wählt daher ein entsprechendes äußeres Erkennungszeichen, einen manierierten Sprachstil oder gar eine verstiegene politische Idee (Franz Werfel). Walter Benjamin etwa erkennt in dieser Haltung „die konsequente, organisierte, gestählte Betrachtung des Daseins vom chemisch-reinen Konsumentenstandpunk ${ }^{\text {" }}$. Dabei stellt Bourdieu gerade an den Produkten der industriellen Massenkultur ein Phänomen fest, das ihn von einem „paradoxen Snobismus“ sprechen lässt. Dieser hefte sich an Gegenstände, die im Prinzip zugänglich seien als wertvolle Kulturgüter - „der Konsum dieser Produkte setzt weniger kulturelles Kapital voraus“ ", deren Besitz indes durchaus eine distinktive Funktion erfülle. Gerade die sogenannte Popkultur gründet auf diesem Paradox ${ }^{8}$. Dem entspricht auf der Ebene des Politischen durchaus die von Frank Degeler und Ute Paulokat vorgenommene Analyse der Popliteratur als

ein wohlbekannte[s] Phänomen einer Abgrenzungsbemühung gegenüber einer (stark politisierten) Vorgängergeneration [...], welche [...] schon alles erkämpft hat, wofür es sich zu kämpfen lohnt, und vor lauter Verständnis, offenheit und Toleranz ihrer Nachfolge-Generation keinerlei Reibefläche bietet. Als einzige Protestform bleibt lediglich ein Rückzug ins Neo-Konservative oder A-Politische9.

Überdies sei die Popliteratur vor allem ein „Ausdruck einer neuen Jugendkultur“. Durch die Darstellung von jugendlichen Protagonisten in einer popkulturell bestimmten Welt biete diese Literatur dem „gleichaltrigen Publikum [perfekte] Identifikationsmöglichkeiten ${ }^{\text {“10 }}$. So erhebt die Popkultur mit ihrer Freude am Zitieren, mit der plakativ vorgetragenen Leichtigkeit des Seins, die den Dilettantismus über einen akademisierten Kulturbetrieb setzt, den von Benjamin den „chemisch-reinen Konsumentenstandpunkt" zur einzig verbindlichen Lebensweisheit.

5 Ein wesentliches Merkmal der neuen Popliteratur ist die Verwendung von „Popmusik als Thema und Formatvorlage der Literatur ${ }^{\text {“11 }}$. Songtitel und Zitate von Songs spielen in den Texten eine konkrete Rolle, einerseits als strukturierende Elemente der Erzählung, als Leitmotive, die den Text rhythmisieren. Andererseits sind Musiktitel Bestandteil des Archivs, das durch die Erzählung erstellt wird. ${ }^{12} \mathrm{Zu}$ dem Archiv gehören darüber hinaus noch Markennamen von Objekten des täglichen Lebens, Werbespots, aber auch 
audiovisuelle Medien, derer sich der Erzähler bedient. Diese „audiovisuelle[n] Medien schaffen ein kollektives Wissen, auf das man sich beziehen kann. ${ }^{13}$ Objekte genauso wie „Lese-, Musik- und Mediengewohnheiten“ erlauben es, Rückschlüsse auf eine Person zu ziehen; Film- und Musiktitel sagen etwas über die Befindlichkeit der Protagonisten aus. Der von ihnen geschilderte Alltag charakterisiere sich gewöhnlich durch „Zweifel, Hoffnungslosigkeit, Sehnsüchte, Perspektiv- und Orientierungslosigkeit sowie vor allem Angst vor der Zukunft" ${ }^{14}$ : Die Konsequenz ist der allmähliche Rückzug ins Private. Die Charakteristika dieser Literatur und die Haltung, der sie entspringt, sollen am Beispiel von Benjamin Stuckrad-Barres Roman Soloalbum dargestellt werden.

6 Soloalbum ${ }^{15}$ ist der erste Roman Stuckrad-Barres, der schon in seiner Schulzeit als freier Autor geschrieben hat. Er ist im Jahre 1975 in Bremen als Sohn eines Pastors geboren, verbringt seine Jugend aber größtenteils in Göttingen; nach dem Abitur studiert er kurze Zeit (wenige Wochen) Germanistik, bricht das Studium aber sehr bald $\mathrm{ab}$ und arbeitet unter anderem als Redakteur bei der Musikzeitschrift Rolling Stone, bei dem Plattenlabel Motor Music und als Autor der „Harald Schmidt Show“. Außerdem schreibt er als freier Mitarbeiter Feuilletons für verschiedene Tageszeitungen (taz, FAZ, Stern). Eine Auswahl seiner journalistischen Arbeiten liegt unter dem Titel Remix (1999) bzw. Remix 2 (2004) vor. Nach Soloalbum (1998), veröffentlicht Stuckrad-Barre im Jahresrhythmus die Romane Livealbum (1999), Blackbox (2000) und Transskript (2001). Es folgen Was. Wir. Wissen. (2006) und die Reportagensammlung Auch Deutsche unter den Opfern (2010). Inzwischen verfasst er seine Feuilletons ausschließlich für die Zeitungen des Springer-Verlags (Bild, Welt am Sonntag). Seit 2010 moderiert er auf dem Sender ZDF Neo die TalkShow Stuckrad Late Night Show.

7 Wie der Titel Soloalbum bereits andeutet, spielt Popmusik eine wesentliche Rolle in dem Roman, der wie eine LP aufgebaut ist, indem er in zwei Teile ,zerfällt', eine Vorderund eine Rückseite. Die einzelnen Kapitel tragen alle Titel von Liedern der englischen Rockgruppe „Oasis“, einer Vertreterin des sogenannten „Britpop“, die der Erzähler ganz besonders schätzt : „Oasis [...] ja die Größten, das sagen wir, das sagen die“. (243)

8 Hauptfigur des Romans ist ein namenloser, in Hamburg lebender Ich-Erzähler, der von seiner langjährigen Freundin Katharina verlassen wird. Sie beendet die Beziehung ganz dem Zeitgeist entsprechend, indem sie ihm die Trennung durch ein Fax mitteilt. Der Erzähler empfindet diese Trennung als äußerst schmerzhaft. Alle Versuche, Katharina wieder zu erobern, schlagen fehl. Seine Anrufe und Geburtstagsgeschenke erweisen sich eher noch als kontraproduktiv. Auch die räumliche Entfernung - Katharina übersiedelt nach Passau - hilft ihm nicht, über die Trennung hinwegzukommen, ebensowenig die Tatsache, dass sie einen neuen Lebenspartner hat.

Alle Versuche des Erzählers, eine neue Freundin zu finden, schlagen fehl, weil er seine Initiativen immer nach kurzer Zeit selbst zum Scheitern bringt: Er bleibt seinen Verabredungen fern, weshalb einmal eine sitzen gelassene Frau sogar die Polizei seine Wohnung aufbrechen lässt, aus Angst, es sei ihm etwas zugestoßen. Mit einer anderen Frau zieht er zusammen, merkt aber nach wenigen Tagen des Zusammenlebens, dass sie nicht nach seinem Geschmack ist und beendet die Beziehung. Er geht auf Partys, 
kokst gelegentlich, doch diese Abwechslungen lassen ihn Katharina nicht vergessen. Vielmehr zieht er sich in seine Wohnung zurück, die immer mehr verkommt, ist mit seinem Aussehen nicht mehr zufrieden, geht joggen, verordnet sich einen strengen Diät- und Fitnessplan und gibt dann gleich wieder Anfällen von Bulimie nach. Am Ende des Romans verlässt er Hamburg, tritt in einer anderen Stadt eine neue Stelle an. Auch in der neuen Umgebung zieht er durch die Bars, trinkt, kokst und versucht eine neue Freundin zu finden, doch hier kommt er allmählich von Katharina los.

Der Roman beschreibt das Lebensgefühl eines jungen Mannes in den neunziger Jahren des vorigen Jahrhunderts, das durch die bereits zitierte Orientierungs- und Perspektivlosigkeit gekennzeichnet ist. ${ }^{16}$ Wie viele andere seiner Generation zieht sich der Erzähler in ein Universum aus Popliedern, Filmen und Drogen zurück und kommentiert sein Handeln mit Filmszenen. Dabei vereinsamt er zusehends :

Kennt man ja aus Filmen : Du bist allein, alleingelassen worden, genauer gesagt, und alle deine Freunde, wenn da noch welche sind, bemuttern dich [...] zum Glück habe ich keine Freunde. Also nicht dieses ganz Üble, einen Freundeskreis. Da hätte ich ja schon lieber einen Mitbewohner oder eine Haushaltskasse, ein Etagenklo oder einen Fernsehraum. Eine Mitfahrgelegenheit, was auch immer. (37)

11 Von der Literaturkritik wird den Autoren des Popromans immer wieder vorgeworfen, dass in ihren Romanen keine exakte Trennung zwischen Autor und Erzähler zu erkennen sei. Der Autor Stuckrad-Barre selbst dagegen will nicht mit dem Erzähler seines Romans identifiziert werden und als lebendes Beispiel dieses Lebensgefühls gelten. Als der Hamburger Journalist Ulrich Hoffman nach der Lektüre von Soloalbum mit Stuckrad-Barre eine Interview über Drogenkonsum machen will, "weil der Held in ,Soloalbum' dem Drogenkonsum ja zumindest nicht abgeneigt ist" (248), und der Journalist sich die Fragen stellt, ob der Roman nicht drogenverherrlichend sei, bzw. als Symbol für ein Lebensgefühl stehe, entgegnet der Autor, dass er „als Symbol für ein Lebensgefühl nicht zur Verfügung“ stehe und sich der Interviewer lieber die Platte This ist Hardcore von Pulp kaufen solle.

Der Begriff „Pop“ in Stuckrad-Barres Pop-Roman reduziert seine Bedeutung nicht auf die Abkürzung pop im Sinne von populär, also das breite Publikum betreffend. Vielmehr ist die neue Popliteratur auf ein jugendliches Leserzielpublikum ausgerichtet, das, gefangen in einer Medienwelt, in der der Konsum eben dieser Medien auf Kosten der direkten zwischenmenschlichen Beziehungen stetig zunimmt und damit einen ausgeprägten Egozentrismus fördert, in dem der Erzähler ganz aufgeht.

Der Ich-Erzähler im Roman Soloalbum agiert als Snob, da sein Hauptanliegen darin besteht, seine Andersartigkeit und sein Überlegenheitsgefühl zu betonen und sich so von seiner Umwelt zu differenzieren. Er hat zwar kurz in einer Wohngemeinschaft gewohnt, hat es dabei aber immer vermieden, mit den anderen Mitbewohnern in engeren Kontakt zu kommen :

Ich habe nie mit ihnen gekocht und gespielt und ferngesehen, nie geputzt und nie eingekauft. Ich ging in diesem Haus nur pissen (im Sitzen) und habe dort geschlafen und gelesen, mehr wollte ich von dieser Heimstatt, die bloß Schlafstatt war, nicht. (89)

14 In der Bemerkung des Ich-Erzählers, dass er in seine Wohnung nur zum „Pissen“ gekommen sei und alle Verpflichtungen, die das Wohnen in einer Gemeinschaft mit 
sich bringt, ignoriert habe, spiegelt sich die bewusste Abgrenzung von den Mitbewohnern, ja seine ganze Verachtung ihnen gegenüber wieder.

Die Verhaltensweise des Erzählers den Frauen gegenüber unterscheidet sich in nichts vom Umgang mit den Mitbewohnern der Wohngemeinschaft. Der nach der Trennung von seiner Freundin Katharina Vereinsamte „hat große Schwierigkeiten, den Betrieb hier aufrechtzuerhalten“ (15). Um sich vor Enttäuschungen zu schützen, begräbt er alle Erwartungen an den Alltag, erwartet „keine Post mehr, keine Anrufe, und [... tut] gut daran, denn es kommt ja auch nichts.“ (136) Trotzdem ist er ständig auf der Suche nach einem Ersatz für Katharina, findet aber nur Nachfolgerinnen, die seinen hohen Ansprüchen nicht entsprechen und für die er nur kritische Kommentare übrig hat. Kurze Zeit lebt er mit Nadja zusammen, zu der ihm nichts anderes einfällt als : „Wenn man das D in der Mitte wegläßt, heißt sie Naja. Da denke ich jetzt mal nicht zu lange darüber nach.“ (121) Als einzigen Grund, weshalb er dennoch mit ihr kurze Zeit zusammen bleibt, führt er seinen sexuellen Notstand an, aber selbst über die erotische Beziehung äußert er sich abschätzig :

[...] in sehr starken oder sehr schwachen Momenten möchte ich sie sofort loswerden. Dann denk ich ans Ficken. Das Ficken mir ihr macht nicht sonderlich Spaß, aber der wenige Spaß ist angesichts der monatelangen Totalflaute natürlich immer noch kosmisch. (181)

Obwohl der Erzähler selbst vor allem aus sexuellen Gründen Kontakt zum anderen Geschlecht sucht, ist er von jenen Zeitschriften angeekelt, in denen Männer und Frauen Anzeigen für sexuelle Kontakte aufgeben, ihre Absichten aber hinter allgemeinen Formulierungen verstecken. Es sind Annoncen
mit Foto und hirnamputierten Antworten auf nicht weniger hirnamputierte Fragen. Sind Sie mit ihrem Gehalt zufrieden? Sonntagsfrühstück im Bett: Was gibt es bei Ihnen? Wie oft pro Woche/Tag hätten Sie gerne Sex? Beschreiben Sei eine ideale Nacht. Lustig ist, daß die Männer nie schreiben, was ihr eigentliches Motiv für diese Verzweiflungstat, sich da abbilden zu lassen (FICKEN), sondern immer, lange reden und kuscheln, viel Zeit haben füreinander' [...] Frauen [...] geben dann präzise an, was die Männer meinen : ,Liebe machen'. (215-216)

17 Als Alternative zur Kontaktaufnahme mittels Anzeigen schlägt er zehn „Singles zum Verlieben“ von „Oasis“ vor, die sich als Mittel zur Anbahnung von erotischen Beziehungen eignen sollen.

In seiner Zurückgezogenheit intensiviert sich der Medienkonsum des Erzählers. Sein Musikgeschmack ist bestimmt von A-priori-Urteilen, wobei er wiederum lediglich an der Gruppe „Oasis“ Gefallen findet. Ihre neue Platte „,Stand byme' ist eher eine schlechte Oasis-Singel, aber natürlich eine gute Single, weil ja von Oasis.“ (231) Bei den Fernsehprogrammen verabscheut er Talk-Shows, in denen sich sehr junge Teilnehmer produzieren und über langweilige Wirtschaftsthemen diskutieren (223) ; besonders zuwider sind ihm Fernsehsender mit Programmen, zu denen er nicht gehört, und darunter wiederum vor allem solche, die sich als Sender der spießigen Kleinbürger etabliert haben :

Ja, der MDR, der Durchhaltesender von und für drüben mit seinen abstrusen Volksmusiksendungen und Familienserien für all die Arbeitslosen. Irgendeine Gala wird da übertragen, klar stehen die Ossis drauf, lauter beliebige Preise für beliebige Prominente. Und dann Arschkriechreden. Danke auch an meine Mutter. Und Variationen über die Sätze :

- Ich bin kein großer Redner. [...]

- Große Worte sind nicht mein Ding. 
- Ich danke den Juroren, vor allem aber meinem Publikum, das mir den Luxus erlaubt hat, mein Hobby zum Beruf gemacht zu haben. (126)

In einem Interview mit Britta Kuck erklärt Benjamin von Stuckrad-Barre, dass es sich in seinem Roman Soloalbum „um ihn“ handle. ${ }^{17}$ Mit der Publikation dieses Romans ist ihm zweifellos ein Bestseller gelungen, und obwohl er in einem Interview behauptet, Verkaufszahlen würden ihn nicht „stärken“" ${ }^{18}$, sind es nicht zuletzt diese Verkaufszahlen, die ihn nach dem Urteil einiger Literaturkritiker zum „neuen Messias der Popliteratur" ${ }^{{ }_{19} 9}$ gemacht haben. Der kommerzielle Erfolg dieses und seiner nachfolgenden Romane ist auch der Grund, weshalb er im Fernsehen seine eigene Sendung bekommen hat: Seit 2010 moderiert er auf dem Extrakanal ZDF Neo die Stuckrad Late Night Show, eine Talk-Show, die sich von ihrer Sendezeit (22.25) deutlich von den zu den besten Sendezeiten ausgestrahlten populären Talkshows der großen Sender unterscheidet. Stuckrad-Barre hat in Deutschland mit seinem literarischen Schaffen genauso wie mit seinen Fernsehauftritten in Nischenprogrammen für eine bestimmte Zielgruppe den Status eines Pop-Stars erreicht 
und hebt sich somit von anderen Autoren, vor allem von Autoren von literarischem Rang ab. Die Attitüde steht bei ihm - wie bei jedem Snob - über dem Werk.

\section{NOTES}

1. Siehe dazu : Moritz Baßler, Der deutsche Pop-Roman. Die neuen Archivisten, München : Verlag C. H. Beck, 2002.

2. Ibid., p. 13.

3. Unter dem Stichwort „Snob“ ist im Oxford English Dictionary (OED) folgende Definition zu lesen : Ein Snob sei ,jemand, der diejenigen verachtet, die er in Bezug auf den Rang, die Leistungen oder die Klasse als tieferstehend betrachtet.“ Ursprünglich hatte der Begriff „Snob“ aber eine ganz andere Bedeutung: Snob war, nach dem OED, ein Ausdruck der Studentensprache in Cambridge. Der Begriff bezeichnete Personen aus der Mittel- und Unterschicht, die „wenig oder keine Erziehung" genossen und keinen guten (der Oberschicht entsprechenden) Geschmack hatten. Die Bedeutungsänderung des Begriffs geht vor allem auf den viktorianischen Romancier William Makepeace Thackeray (1811-1863) zurück, der in den Jahren 1847/1848 unter dem Namen „Mr. Snob" in der satirischen Wochenschrift Punch Artikel veröffentlichte, in denen er darüber berichtet, zu welchen Auswüchsen es durch den Versuch der Mittelschicht, den Adel in ihrem Geschmack und in ihren Manieren zu imitieren, gekommen ist. Das beginnende neunzehnte Jahrhundert ist eine Epoche, in der sich der englische Mittelstand konsolidiert und danach trachtet, durch sein öffentliches Auftreten und seine Erscheinung, bei dem Modereferenzen eine dominante Rolle spielen, es der Oberschicht gleichzutun, ja sie zu übertreffen, wobei der gute Geschmack oft auf der Strecke geblieben ist. Diese satirischen Texte veröffentlicht Thackeray schließlich in dem Buch The Books of Snobs (auf Deutsch erstmals 1851 unter dem Titel Die Snobs. Humoristische Bilder aus Alt-England, es folgen weitere Übersetzungen bzw. Auflagen unter den Titeln Das Snobsbuch, Das Snob-Buch und Das Buch der Snobs) Siehe dazu : Sean Latham, Am I a Snob? Modernism and the Novel.New York : Cornell University Press, 2003, p. 11-18.

4. "James I. war ein Snob und ein schottischer Snob, in der Welt keine aggressiveren Lebewesen“ Latham, Snob, p. 18. "James I was a Snob, and a Scotch Snob, than which the world contains no more offensive creature."

5. Zur gleichen Zeit veröffentlichte Thackeray auch seinen Roman Jahrmarkt der Eitelkeiten (Vanity Fair, 1847-48), der ebenfalls die Thematik der aufstrebenden, sich emanzipierenden Mittelklasse behandelt.

6. Latham, Snob, p. 16, „The snob can be alternately haughty, brutal, stupid and perfectly selfconfident or poor, wondering, kneeling, [and] simple“.

7. Walter Benjamin, „Zum Bilde Prousts“ (1929), in: Gesammelte Schriften 2/1., hg. von R. Tiedemann, Frankfurt a. M. : Suhrkamp , 1977, p. 319.

8. Pierre Bourdieu, „Kultur in Gefahr“, in : Gegenfeuer 2, Konstanz : uvk, 2001, p. 82-99, hier p. 85.

9. Frank Degler/Ute Paulokat, Neue Deutsche Popliteratur, Paderborn : Wilhelm Fink Verlag, 2008,

p. 11.

10. Ibid., p. 11.

11. Ibid., p. 9.

12. Siehe Baßler, Pop-Roman, p. 9-45.

13. Degler/Paulokat, Neue Deutsche Popliteratur, p. 10. 
14. Ibid., p. 11.

15. Benjamin von Stuckrad-Barre, Soloalbum, 7. Auflage, Köln : Kiepenheuer \& Witsch, 2009. In der Folge werden Zitate aus dem Roman durch die Angabe der in Klammern gesetzten Seitenzahl angegeben.

16. In Frankreich hat Frédéric Beigbeder einen ähnlichen Protagonisten entworfen in dem Roman : Mémoires d'un jeune homme dérangé, Paris, La Table Ronde, 1990.

17. Interview Britta Kucks mit Benjamin von Stuckrad Barre :

http://www.my-culture.de/Buch\&Co/StuckradBarre_Interview.htm (05. 10. 2011).

18. Cf. Britta Kuck.

19. Daniel Müller, Stuckrad-Barre: „Der Künstler darf sich wehtun“, Interview mit Benjamin von Stuckrad-Barre, in : Machtmaschine, 1. 12. 2008.

\section{RÉSUMÉS}

In seinem ersten Roman Soloalbum stellt Benjamin von Stuckrad-Barre das Lebensgefühl der Generation der Zwanzigjährigen im Deutschland der 1990er Jahre dar, das sich durch eine apolitische Grundhaltung und den Rückzug ins Private charakterisiert. Hauptfigur ist ein nicht näher benannter Erzähler, der sein Dasein in eine „Rhetorik der Ausgrenzung“ kleidet, um den Weltschmerz zu artikulieren, nachdem ihn seine Freundin Katharina verlassen hat. Alle seine Versuche, eine neue Freundin $\mathrm{zu}$ finden und so über diese Trennung hinweg zu kommen, scheitern nicht zuletzt an seiner snobistischen Haltung den Freunden und Kollegen gegenüber, sodass er - immer stärker isoliert - nur in der Musik der Pop-Gruppe „Oasis“ eine Antwort auf seine Misere findet. Der Autor hat mit dem Roman den Status eines Pop-Stars erreicht. Von seinen Schriftstellerkollegen grenzt er sich dadurch ab, dass er eine Fernseh-Talkshow im Spätabendprogramm moderiert, was ihn zum Mittelpunkt einer snobistisch zu nennenden Fangemeinde macht, die ganz im Bann seines plakativ vorgetragenen Egoismus zu stehen scheint.

Soloalbum, roman de Benjamin von Stuckrad-Barre, décrit la façon de vivre des jeunes adultes dans les années quatre-vingt-dix du xxe siècle en Allemagne. Cette génération apolitique vit repliée sur elle-même. Le narrateur est le personnage principal du roman. Il n'a pas de nom. Il décrit dans une langue marquée par une «rhétorique d'exclusion » la vie qu'il mène après avoir été quitté par son amie Katharina. Toutes ses tentatives pour accepter son départ et la remplacer échouent à cause de son attitude snob vis-à-vis de son entourage, de sorte qu'il se trouve de plus en plus isolé et qu'il ne lui reste plus que la musique du groupe « Oasis ». Le roman de StuckradBarre a eu beaucoup de succès et l'auteur est devenu une sorte de pop-star littéraire. Par ailleurs, il s'est démarqué de ses collègues écrivains en animant une émission-débat à la télévision. Cette activité lui vaut d'avoir beaucoup de fans - fascinés par la mise en scène d'un égoïsme hypertrophié.

Benjamin von Stuckrad-Barre shows in his novel Soloalbum the attitude towards life of the German young generation in the nineties. This generation is mainly apolitical and retreated to privacy. The main character of the novel is an unnamed narrator using a language which can be qualified as "rhetoric of exclusion" - after having been left by his girlfriend Katharina. Every attempt to deal with this situation and to find a new girlfriend is a failure because of his snobbish 
behaviour towards his friends and colleagues. His only rescue is the music of the pop-group "Oasis". The novel of Stuckrad-Barre was a real success in Germany and the author became a pop star of literature. In addition to this he is the talk-master of a late night show on German TV to distinguish himself from other writers. And his snobbish fans are fascinated by his outstanding ego.

INDEX

Mots-clés : popliteratur

oeuvrecitee Soloalbum, The Books of Snobs

\section{AUTEURS}

ALFRED STRASSER

Université Charles-de-Gaulle - Lille 3 